\title{
Level-Set-Based Artery-Vein Separation in Blood Pool Agent CE-MR Angiograms
}

\author{
Cornelis M. van Bemmel*, Luuk J. Spreeuwers, Max A. Viergever, Member, IEEE, and \\ Wiro J. Niessen, Associate Member, IEEE
}

\begin{abstract}
Blood pool agents (BPAs) for contrast-enhanced (CE) magnetic-resonance angiography (MRA) allow prolonged imaging times for higher contrast and resolution. Imaging is performed during the steady state when the contrast agent is distributed through the complete vascular system. However, simultaneous venous and arterial enhancement in this steady state hampers interpretation. In order to improve visualization of the arteries and veins from steady-state BPA data, a semiautomated method for artery-vein separation is presented. In this method, the central arterial axis and central venous axis are used as initializations for two surfaces that simultaneously evolve in order to capture the arterial and venous parts of the vasculature using the level-set framework. Since arteries and veins can be in close proximity of each other, leakage from the evolving arterial (venous) surface into the venous (arterial) part of the vasculature is inevitable. In these situations, voxels are labeled arterial or venous based on the arrival time of the respective surface. The evolution is steered by external forces related to feature images derived from the image data and by internal forces related to the geometry of the level sets. In this paper, the robustness and accuracy of three external forces (based on image intensity, image gradient, and vessel-enhancement filtering) and combinations of them are investigated and tested on seven patient datasets. To this end, results with the level-set-based segmentation are compared to the reference-standard manually obtained segmentations. Best results are achieved by applying a combination of intensity- and gradient-based forces and a smoothness constraint based on the curvature of the surface. By applying this combination to the seven datasets, it is shown that, with minimal user interaction, artery-vein separation for improved arterial and venous visualization in BPA CE-MRA can be achieved.
\end{abstract}

Index Terms-Artery-vein separation (AV separation), blood pool agent (BPA), contrast-enhanced magentic-resonance angiography (CE-MRA), level set, separation.

\section{INTRODUCTION}

B LOOD POOL agents (BPAs) for contrast-enhanced magentic-resonance angiography (CE-MRA) have a prolonged intra-vascular half-life and provide strong $\mathrm{T} 1$ relaxation even at low concentrations [1]. Therefore, these agents allow imaging in the steady state, thus providing longer time windows

Manuscript received March 19, 2003; revised May 25, 2003. This work was supported by EasyVision Advanced Development, Philips Medical Systems. The Associate Editor responsible for coordinating the review of this paper and recommending its publication was J. Liang. Asterisk indicates corresponding author.

*C. M. van Bemmel is with the University Medical Center, Image Sciences Institute, NL-3584 CX Utrecht, The Netherlands (e-mail: kees@isi.uu.nl).

L. J. Spreeuwers, M. A. Viergever, and W. J. Niessen are with the University Medical Center, Image Sciences Institute, NL-3584 CX Utrecht, The Netherlands (e-mail: luuk@isi.uu.nl; max@isi.uu.nl; wiro@isi.uu.nl).

Digital Object Identifier 10.1109/TMI.2003.817756
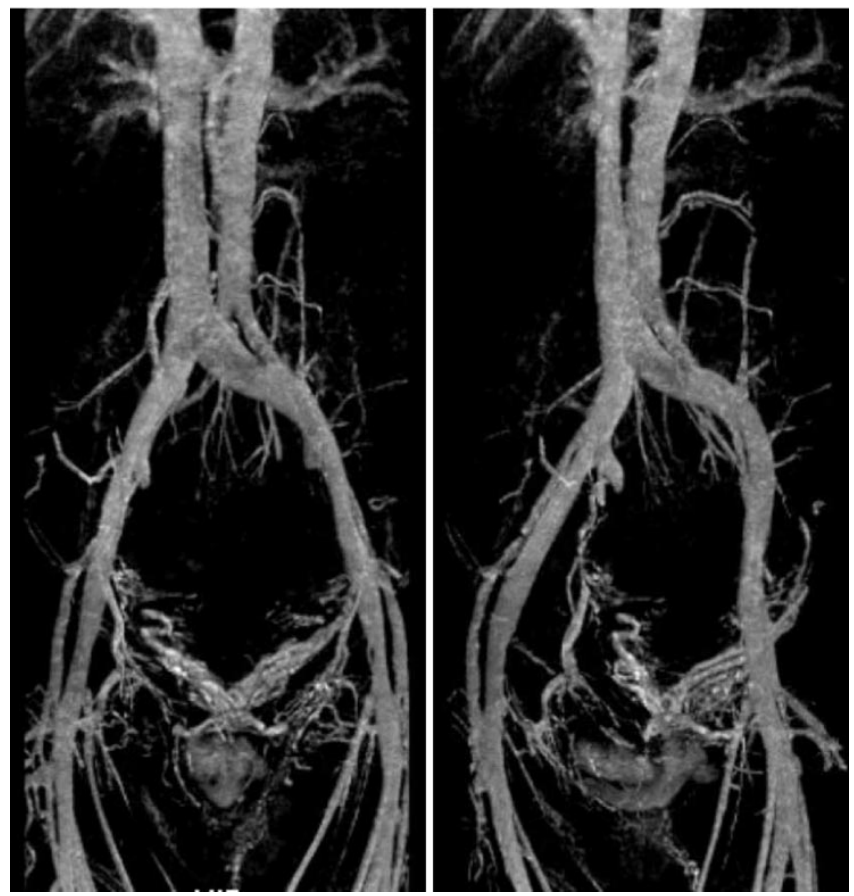

Fig. 1. MIP of a dataset acquired during the steady state (left frame: posteroanterior, right frame: oblique). Simultaneous enhancement of arteries and veins hampers interpretation.

for image acquisition, which can be advantageous if high contrast and/or resolution is required and a large anatomical region needs to be covered [2]. However, an important drawback of imaging in the steady state is the simultaneous enhancement of arteries and veins, which hampers the interpretation of the steady-state data (see Fig. 1).

A method to solve this problem is to separate arteries from veins. Several artery-vein separation (AV separation) techniques have been proposed. Some approaches, e.g., [3]-[7], aim at separating arteries from veins during the acquisition stage. Phase-contrast (PC) techniques [3], [4] are flow dependent and rely on the difference in the blood-flow direction in arteries and veins. Therefore, these techniques are only suitable for situations where the blood-flow direction in arteries and veins is opposite. Svensson et al. separate arteries from veins in the lower extremities based on flow-induced phase effects [6]. The method is based on the requirement that all vessels are oriented parallel to the leg. Smaller vessels do not always fulfill this requirement and are, therefore, handled incorrectly and appear suppressed after segmentation. Also, longitudinal small vessels and larger veins in the lower part of the image volume are 


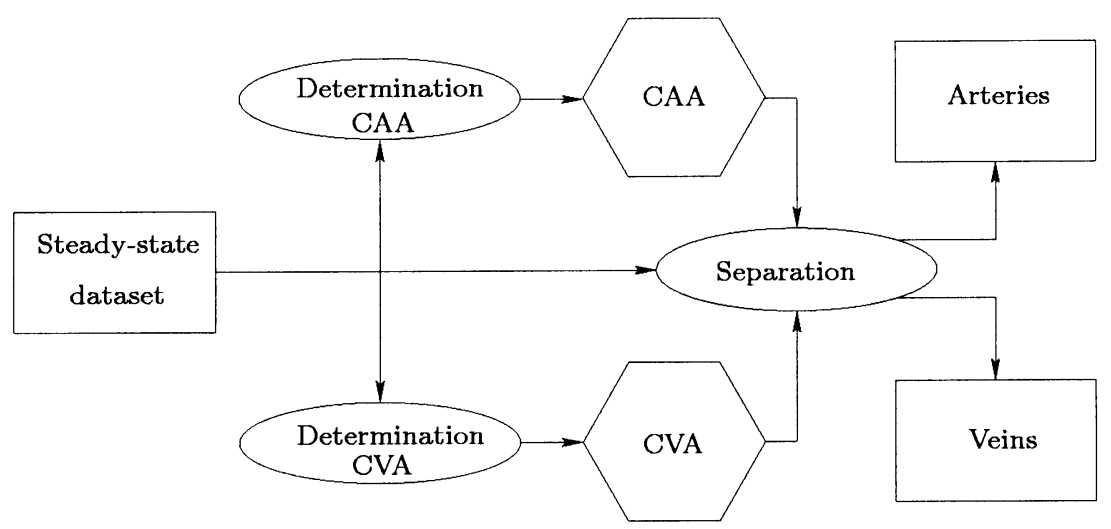

Fig. 2. Block diagram of the proposed method. First, the CAA and CVA are determined. Subsequently, the arteries and veins are separated using level-set techniques with the CAA and CVA as initializations.

hard to successfully segment due to their low flow velocities. Wang et al. discriminate arteries from veins depending on the oxygenation level [5]. This flow-independent method relies on the blood oxygenation level-dependent (BOLD) effect and is limited owing to the fact that the oxygen level in the veins is sometimes higher than expected. Mazaheri et al. characterize each pixel as arterial, venous, or unenhanced background tissue during the first pass of the contrast agent using a time-resolved acquisition scheme [7]. However, the period in which the venous uptake lags the arterial uptake is very critical.

Numerous papers have addressed the issue of MRA vessel visualization and segmentation using post-processing techniques, e.g., [8]-[13]. For a comprehensive overview on magnetic resonance (MR) vascular image processing, we refer to [14] and [15]. The issue of AV separation has only been addressed by a few authors [16]-[20]. Bock et al. propose a method for AV separation based on the difference in temporal enhancement kinetics of arterial and venous vascular signal in three-dimensional (3-D) MR angiograms of the lung [16]. Niessen et al. improve arterial visualization by suppressing the major overlapping veins in maximum-intensity projections (MIPs) [17]. This visualization technique is only applicable for larger overlapping veins. Stefancik and Sonka show the feasibility of AV separation using a graph-search approach [18]. Tizon and Smedby propose an algorithm that uses the gray-scale degree of connectedness to split the original volume into different vessel components [19]. A certain amount of user interaction is needed to label the vessels either arterial or venous. Problems may occur in case of an arterial stenosis since vascular parts distal to the stenosis with respect to the initialization are difficult to capture owing to a low connectedness across the stenosis. The most extensive work on AV separation has been reported by Lei et al. [20] who use the principle of fuzzy connectedness [21]. First, all vascular structures are segmented from the background. Second, arteries are separated within this entire vessel structure from veins via an iterative fuzzy-connectedness procedure. Promising results have been reported on a large number of datasets. However, the authors acknowledge that validation is still required to assure that the results can clinically be used.

In this paper a level-set-based (LSB) method for AV separation is presented, where the central arterial axis (CAA) and the central venous axis (CVA) are used for initializing two level sets that simultaneously capture the arterial and venous vessel structures, respectively. To test the accuracy of our method, we compare the LSB segmentations with the reference standard, manually obtained segmentations.

The novelty of our study is twofold. First, by utilizing a central vessel axis as initialization for the evolving level sets, we assure a good initialization, even if a stenosis is present. Also, the use of the central vessel axis as initialization circumvents the problem that arises when a more localized initialization is used. In that case, oversegmentation will occur in the neighborhood of the initialization, while the vessel distal to the initialization is not yet segmented. Second, our method introduces a simultaneous evolution of level sets so that the arrival time of the respective fronts can be utilized to estimate which part of the dataset belongs to the arterial or venous part of the vasculature, respectively.

\section{METHODS}

The method described in this paper utilizes the CAA and CVA as initializations for LSB separation of the arteries and veins (see Fig. 2). The first step of the procedure is to determine the central axes for initialization of the level-set method (Section II-A). For the central vessel-axis extraction, a feature image is used that enhances vessel-like structures (Section II-B). AV separation is performed using the level-set framework. For both this first step and the level-set implementation, a feature image is used that enhances vessel-like structures. This is discussed in Section II-B. Section II-C deals with the level-set implementation.

\section{A. Extracting Central Axes for Initialization}

The CAA and CVA can be determined manually from the steady-state dataset. However, manual delineation of the CAA and CVA is a tedious procedure. Therefore, a supervised procedure for CAA and CVA delineation has been implemented. In this procedure a minimum-cost path (MCP) is determined between two (or more) user-defined points in the arterial and venous parts of the vasculature, respectively. Hereto, the steadystate dataset is filtered in order to enhance vessel-like structures [10] (see also Section II-B). The reciprocal value of the 
filtered image represents the costs for the MCP algorithm. Subsequently, a bi-directional search tree is started from both the starting node and goal node simultaneously. The evolution of the search tree is continued until the two fronts meet. In the case of more user-defined points, the procedure is continued until all points have been connected. A more detailed description of the MCP algorithm is given in [22]. In a previous study, we have shown that if a first-pass dataset is available in which only the arterial part of the vasculature is enhanced, this step can be carried out with even less user interaction [23]. Here, we have chosen the supervised technique on the steady-state data since a dataset acquired during the first pass of the contrast agent is not always available. Our supervised technique strongly reduces the amount of user interaction required for CAA/CVA delineation.

\section{B. Vessel Enhancement}

Both for extraction of the CAA and CVA, and for separation of the arteries and veins, a feature image is used in which voxels with a high probability to be part of the center of a vascular structure are enhanced. Hereto, a multiscale filter is employed that analyzes the local second-order image structure. In this paper, we only mention the most relevant formulas. For a detailed description, we refer to [10].

The filter depends on the eigenvalues of the Hessian matrix $\lambda_{\sigma, k}(k=1,2,3)$ that correspond to the normalized eigenvectors $\hat{\mathbf{u}}_{\sigma, k}$, i.e., $\left|\lambda_{\sigma, 1}\right| \leq\left|\lambda_{\sigma, 2}\right| \leq\left|\lambda_{\sigma, 3}\right|$. The eigenvectors $\hat{\mathbf{u}}_{\sigma, k}$ compose the three orthonormal directions; in case of a vessel structure, $\hat{\mathbf{u}}_{1}$ indicates the direction along the vessel (minimum intensity variation), and $\hat{\mathbf{u}}_{2}$ and $\hat{\mathbf{u}}_{3}$ form a base for the plane orthogonal to the vessel direction.

From an analysis of the eigenvalues, two geometric ratios $\left(\mathcal{R}_{A}\right.$ and $\left.\mathcal{R}_{B}\right)$ and a measure for distinguishing background voxels from vessel voxels $(\mathcal{S})$ are introduced as follows:

$$
\begin{aligned}
& \mathcal{R}_{A}=\frac{\frac{\text { (radius of largest cross section area) }}{\pi}}{(\text { largest axis semilength })^{2}}=\frac{\left|\lambda_{2}\right|}{\left|\lambda_{3}\right|} \\
& \mathcal{R}_{B}=\frac{\frac{\text { volume }}{\left(\frac{4 \pi}{3}\right)}}{\left(\frac{\text { largest cross section area }}{\pi}\right)^{\frac{3}{2}}}=\frac{\left|\lambda_{1}\right|}{\sqrt{\left|\lambda_{2} \lambda_{3}\right|}} \\
& \mathcal{S} \triangleq\|\mathcal{H}\|_{F}=\sqrt{\sum_{j} \lambda_{j}^{2}}
\end{aligned}
$$

The ratio $\mathcal{R}_{A}$ is essential for distinguishing between plate-like $\left(\mathcal{R}_{A} \ll 1\right)$ and line-like structures $\left(\mathcal{R}_{A} \approx 1\right)$. The ratio $\mathcal{R}_{B}$ (small in case of a line-like structure) accounts for the deviation from a blob-like structure $\left(\mathcal{R}_{B} \approx 1\right)$. $\mathcal{S}$ is a measure of "secondorder structureness," and will be low in the background where no structure is present.

Since, in CE MRA, vessels give higher signals than the background, $\lambda_{2}$ and $\lambda_{3}$ are negative in the case of vessel-like structures. Therefore, the vessel-enhancement filter $\mathcal{V}(x, \sigma)$ at location $x$ and at scale $\sigma$ is defined as

$$
\mathcal{V}(x, \sigma) \triangleq \begin{cases}0, & \text { if } \lambda_{2}>0 \text { or } \lambda_{3}>0 \\ v(x, \sigma), & \text { otherwise }\end{cases}
$$

where

$$
v(x, \sigma)=\left(1-e^{-\frac{\mathcal{R}_{A}^{2}}{2 \alpha^{2}}}\right) e^{-\frac{\mathcal{R}_{\mathcal{B}}^{2}}{2 \beta^{2}}}\left(1-e^{-\frac{S^{2}}{2 \gamma^{2}}}\right) .
$$

The parameters $\alpha, \beta$, and $\gamma$ tune the sensitivity of the filter to deviations in $\mathcal{R}_{A}, \mathcal{R}_{B}$, and $\mathcal{S}$, respectively, relative to the ideal behavior for a line structure. Equation (4) states that the filter response is a function of the scale at which the Gaussian derivatives are computed. The filter is applied at multiple scales that span the range of expected vessel widths according to the imaged anatomy. In order to provide a unique filter output for each voxel, the multiple scale outputs undergo a scale-selection procedure, the maximum filter response across the scales is selected by

$$
\mathcal{V}(x)=\max _{\sigma_{\min } \leq \sigma \leq \sigma_{\max }} \mathcal{V}(x, \sigma)
$$

In this way, different vessel sizes will be detected at their corresponding scales and both small and large vessels will be captured with the same scheme.

\section{LSB AV Separation}

Separation of arteries and veins is achieved via the level-set framework in which the CAA and CVA serve as initializations for two competitive fronts. The separation can be regarded as the evolution of two fronts, or interfaces, toward the boundaries of the arterial and venous vasculature. Rather than evolving an interface itself, in the level-set framework the interface is represented by the zero-level set of a higher dimensional function. To formalize these notions, let $\Gamma(t)$ denote a time-dependent closed $(n-1)$-dimensional hyper-surface, which evolves in its normal direction

$$
\left\{\begin{array}{l}
\frac{\partial \Gamma(t)}{\partial t}=F \cdot \vec{N} \\
\Gamma(0)=\Gamma_{0}
\end{array}\right.
$$

where $F$ denotes the force, $\vec{N}$ denotes the normal vector to the hyper-surface pointing outwards, and $\Gamma_{0}$ denotes the initial hyper-surface.

Now, an $n$-dimensional function $\phi(x, t)$ is defined such that $[\phi(x, t)=0]=\Gamma(t)$, i.e., $\Gamma(t)$ is represented by the zero-level set of $\phi(x, t)$ at all times [24]. It can easily be shown that if $\Gamma(t)$ evolves according to (6), the corresponding evolution of $\phi(x, t)$ is given by

$$
\left\{\begin{array}{l}
\frac{\partial \phi(x, t)}{\partial t}+F|\nabla \phi(x, t)|=0 \\
\phi(x, 0)=\Gamma_{0} .
\end{array}\right.
$$

Thus, in LSB image segmentation, the evolution of $\Gamma(t)$ is implicitly defined by evolving $\phi(x, t)$. This approach has the advantage that topological changes in $\Gamma(t)$ are handled naturally.

The force $F$ can depend on external properties derived from the image to be segmented and intrinsic geometrical properties that can easily be determined, e.g., the curvature to the front $(\kappa=\nabla(\nabla \phi /|\nabla \phi|))$. This is similar to the classical snake segmentation in which an image-based term to capture the object of 
interest and an internal geometry term, to assure smoothness of the obtained segmentation, are used [25]. In the level-set framework, this leads to the following general evolution equation:

$$
\frac{\partial \phi(x, t)}{\partial t}+F_{\text {ext }}\left(1-\epsilon F_{\kappa}\right)|\nabla \phi(x, t)|=0 .
$$

Here, $F_{\text {ext }}$ denotes the external force that is derived from image information, $F_{\kappa}$ is a curvature-based geometric smoothing term, and $\epsilon$ is a weighting factor. A surface has two principal curvatures, i.e., $\kappa_{\min }$ and $\kappa_{\max }$. Since, for tubular structures, the curvature in the plane transversal to the vessel is always large, only the curvature in the longitudinal direction of the vessel, i.e., $F_{\kappa}=\kappa_{\min }$, is weighted to obtain a smooth segmentation in the direction of the vessel axis [11], [26]. To properly capture the vessel boundaries, an appropriate function $F_{\text {ext }}$ and a proper weighting of the image-based and intrinsic force need to be determined. Traditionally, image gradients are used to guide deformable models to object boundaries [27]. However, they are sensitive to noise, and outliers in the data can significantly influence the result. A possible solution to this problem is the use of additional image-based information. For our application, the influence of three image-based forces (and combinations of them) is investigated. Thus, $F_{\text {ext }}$ is composed of one or more of the terms that are discussed below.

The first term is based on intensity information $\left(F_{\text {int }}\right)$. The histogram of the steady-state dataset shows two distinct peaks representing the background and the vasculature, respectively. Two normal distributions are fitted to the histogram of the steady-state dataset using an expectation maximization algorithm [28]. The distributions of the background and vasculature are described by $\mathcal{N}\left(\mu_{b}, \sigma_{b}\right)$ and $\mathcal{N}\left(\mu_{v}, \sigma_{v}\right)$, respectively. Since, in CE-MRA, vessels give higher signal than the background, it is clear that $\mu_{v}>\mu_{b}$. Based on these parameters, the intensity-based force is defined as

$$
F_{\text {int }}(i)=\frac{F_{v}(i)-F_{b}(i)}{F_{v}(i)+F_{b}(i)}
$$

where $i$ is the image grey value, and $F_{b}(i)$ and $F_{v}(i)$ are given by

$$
F_{b}(i)=\frac{1}{\sigma_{b} \sqrt{2 \pi}} \int_{i}^{I_{\max }} e^{-\frac{1}{2}\left(\frac{\zeta-\mu_{b}}{\sigma_{b}}\right)^{2}} d \zeta
$$

and

$$
F_{v}(i)=\frac{1}{\sigma_{v} \sqrt{2 \pi}} \int_{0}^{i} e^{-\frac{1}{2}\left(\frac{\zeta-\mu_{v}}{\sigma_{v}}\right)^{2}} d \zeta .
$$

In (9a), $I_{\max }$ is the maximum grey value in the image. The functions $F_{b}(i)$ and $F_{v}(i)$ describe the probability that a voxel with intensity $i$ belongs to the background and vasculature, respectively. If the probability of a voxel being part of the vasculature is equal to probability of being part of the background, $F_{\text {int }}=0$. As can be seen from (9), $F_{\text {int }}$ ranges $[-1,+1]$. Note that no ad hoc parameter settings are introduced as the values are given by $\left(\mu_{b}, \sigma_{b}\right)$ and $\left(\mu_{v}, \sigma_{v}\right)$.
The second term is based on gradient information $\left(F_{\text {grad }}\right)$. The gradient image is computed by convolving the steady-state dataset with the first-order derivative of a Gaussian kernel

$$
F_{\text {grad }}\left(x, \sigma_{\text {grad }}, c_{\text {grad }}\right)=e^{-\left(\frac{\nabla G_{\sigma_{\text {grad }}} * I(x)}{c_{\text {grad }}}\right)^{2}} .
$$

where $c_{\text {grad }}$ weighs the magnitude of the image gradient obtained by convolving the image $I(x)$ with the first-order derivative Gaussian smoothing filter $\nabla G_{\sigma_{\text {grad }}}$ whose characteristic width is $\sigma_{\text {grad }}$. Note that this gradient-based is strictly positive (range $[0,1]$ ). Therefore, in case of leakage through the vessel wall, the front will continue to grow if only gradient information is used.

The vesselness function, see (5), is maximal at the center of the vessel and decreases to zero at the vessel boundaries [10], making it suitable to be used as the third term that is considered for the image-based force

$$
F_{\text {vessel }}(x)=\mathcal{V}(x) .
$$

The disadvantage of this function is that it falls short if the assumption of tubular structures no longer holds, e.g., at bifurcations. Values of $F_{\text {vessel }}$ range $[0,1]$.

Since the separate terms have different properties, $F_{\text {ext }}$ is composed by multiplying one or more of these terms as follows:

$$
F_{\text {ext }}=F_{\text {int }} F_{\text {grad }} F_{\text {vessel }}
$$

where a term is set to 1 if it is not included. We emphasize that values of $F_{\text {int }}$ range $[-1,+1]$, and values of $F_{\text {grad }}$ and $F_{\text {vessel }}$ range $[0,1]$. Therefore, it is not possible that negative terms cancel out due to the multiplication of different terms. Note that if only $F_{\text {grad }}$ and/or $F_{\text {vessel }}$ are/is applied, there is only an expanding external force, in contrast to applying (a combination with) $F_{\text {int }}$, which makes contraction possible as well. In regions where arteries and veins are in close proximity of each other, leakage from the evolving arterial (venous) front into the venous (arterial) part of the vasculature is inevitable. In these situations, separation can still be achieved based on the recorded passage time of the respective evolving fronts. It is to be expected that the passage time of the zero-level set for a voxel belonging to the arterial (venous) part of the vasculature is smaller for the evolution starting from the CAA (CVA) than starting from the CVA (CAA). Therefore, voxels are labeled arterial or venous based on the arrival time of the respective front. This is referred to as the arrival-time correction. Thus, during the evolution of the two fronts, the arterial front can grow in the venous part of the vasculature (and vice versa), but here is corrected for retrospectively using the recorded passage time.

\section{EXPERIMENTS}

\section{A. Image Acquisition}

For this study, datasets of seven patients are examined. The datasets were acquired during a phase-II study of BPA NC100150 (Nycomed Imaging AS, Oslo, Norway). Informed consent was obtained. Images were obtained during the steady state, in which the BPA is distributed through the entire vasculature and both arteries and veins are enhanced. The images were acquired on a 1.5-T MR imaging system 
(Gyroscan ACS-NT, Philips Medical Systems, Best, The Netherlands). The images were T1-weighted using a fast 3-D gradient echo sequence with the following scan parameters: $\mathrm{TR} / \mathrm{TE}=5.8 / 1.6 \mathrm{~ms}$, flip angle $(\mathrm{FA})=30^{\circ}$. Field of view (FOV) was $450 \times 450 \times 120 \mathrm{~mm}^{3}$. The voxel size of the images is $0.9 \times 0.9 \times 1.0 \mathrm{~mm}^{3}$.

\section{B. Path Tracking}

For the semiautomated path tracking, the vesselness image of the steady-state dataset is computed at 25 scales (exponentially increasing, $\sigma=0.4-13.0 \mathrm{~mm}$ ), after which the MCP is determined. The influence of the parameters $\alpha, \beta$, and $\gamma$ on the accuracy of the central vessel axis determination was determined in a previous study [23]. Best results are obtained for $\alpha$, $\beta=0.25$, and $\gamma / I_{\max }=0.375$, where $I_{\max }$ is the maximum grey value in the image. The CAA and CVA are determined from the steady-state dataset in a supervised way by selecting a few points as initialization for the determination of the MCPs.

\section{Validation Strategy}

To determine the performance of the method, the following strategy is followed. To find the best performing function $F_{\text {ext }}^{*}$ and weighting factor $\epsilon^{*}$ of the curvature-based smoothing term for the LSB segmentation task, the influence of the separate forces and the curvature term are tested. To this end, segmentations obtained with the LSB method are compared to the reference standard as a function of the iteration time $t$ of the evolving fronts. Owing to the enhancement of the entire vascular structure in the steady state, it is not feasible to perform a complete manual segmentation of the aortoiliac vasculature. Therefore, six subvolumes are used for which manual segmentation of the arteries is a feasible task. Subvolumes of both the aorta (large vessel) and iliac region (small vessels) are used for evaluation. In the following, the subscripts "lsb" and "man" denote the $L S B$ method and manual, respectively. The superscripts + and - denote the segmented part, i.e., arteries, and background of the manually segmented image volume, respectively. For each subvolume, similarity $s(t)$ is measured after each iteration step and, thus, a function of time $t$. Similarity is defined as the overlap of a segmented volume, normalized by the average volume of the LSB segmentation and reference standard [29]

$$
s(t)=2 \frac{V_{\mathrm{lsb}}(t) \cap V_{\operatorname{man}}^{+}}{V_{\mathrm{lsb}}(t)+V_{\operatorname{man}}^{+}} .
$$

The mean similarity $(S(t))$ is defined as the mean of all six similarity values at iteration time $t$. $S_{\max }$ is the maximum mean similarity during the evolution. For each subvolume, we also performed an accuracy analysis: the true positive fraction $(t p f(t))$ and false positive fraction $(f p f(t))$ are determined according to

$$
\operatorname{tp} f(t)=\frac{V_{\mathrm{lsb}}(t) \cap V_{\operatorname{man}}^{+}}{V_{\operatorname{man}}^{+}}
$$

and

$$
f p f(t)=\frac{V_{\mathrm{lsb}}(t) \cap V_{\mathrm{man}}^{-}}{V_{\operatorname{man}}^{+}}
$$

where $\operatorname{tpf}(t)(f p f(t))$ measures the overlap of the LSB segmentation and the reference standard (background) as a fraction of the reference standard. Note that both $\operatorname{tp} f(t)$ and $f p f(t)$ are functions of the iteration time. TPF $(t)(F P F(t))$ is the mean true (false) positive fraction and is defined as the mean of all six $t p f(t)(f p f(t))$ values at iteration time $t$.

It is clear that we did not perform a receiver operating characteristic (ROC) analysis. In ROC analysis, $f p f$ is defined as the number of falsely classified voxels as a fraction of the background of the image $\left(V_{\operatorname{man}}^{-}\right)$. In our application, the vascular structures to be segmented are relatively small (approximately $1 \%-2 \%$ of the image volume). Therefore, in a ROC analysis, it is to be expected that FPF will be very small and dependent on the background volume, making the proposed accuracy analysis more useful than the classical ROC analysis. Similar to the ROC analysis, we determine the area under the accuracy plot $\left(A_{\mathrm{acc}}\right)$. In order to compare the performance of the different forces with each other with respect to the $\mathrm{AV}$-separation task, $A_{\text {acc }}$ is determined for $F P F \in[0,1]$ since $F P F$ can be larger than 1 . Note that for $F P F=1, T P F$ can be smaller than 1 .

In order to find the best performing function $F_{\text {ext }}^{*}$ and the weighting factor $\epsilon^{*}$ for $F_{\kappa}$, and the cut point $t^{*}$, the following experiments have been conducted.

1) The influence of the arrival-time correction is examined using $F_{\text {ext }}=F_{\text {int }}$ by showing the results with this correction and without this correction, thus simulating the results when a single level set would have been used.

2) Each term is tested separately using the arrival-time correction and without using curvature $(\epsilon=0,(8))$; the parameters of $F_{\text {ext }}=F_{\text {grad }}$ and $F_{\text {ext }}=F_{\text {vessel }}$ are varied independently. Thus, the following terms are tested.

- $F_{\text {ext }}=F_{\text {int }}[\operatorname{see}(9)]$.

- $F_{\text {ext }}=F_{\text {grad }}$, initially with $\sigma_{\text {grad }} \in$ $\{0.75,1.0,1.25,1.5\}$ and $c_{\text {grad }} \in\{8,16,24,32\}$ [see (10)].

- $F_{\text {ext }}=F_{\text {vessel }}$, with $\alpha, \beta \in\{0.25,0.5\}$ and $\gamma / I_{\max } \in\{0.10,0.25,0.375\}$ [see (11)].

3) Combinations of terms are tested in order to determine $F_{\text {ext }}^{*}$. Since it is not feasible to test all possible combinations of forces with different parameter combinations, we tested all possible combinations of the different terms using the best parameter settings as they were found testing the individual terms. Again, curvature is not taken into account.

4) In order to determine $\epsilon^{*}$, the influence of the curvature term is tested by using $F_{\text {ext }}^{*}$ obtained from the previous experiments and varying $\epsilon \in$ $\{0.0125,0.025,0.05,0.10,0.25\}$.

5) Finally, for all the patient datasets, segmentations of the arterial and venous parts of the vasculature that are seeded by the CVA and CAA are obtained by using $F_{\text {ext }}^{*}$ and $\epsilon^{*}$ in the LSB segmentation. From the accuracy plot belonging to the best performing parameters, a cut point $t^{*}$ is selected.

\section{RESULTS}

\section{A. Path Tracking}

To initialize the LSB separation of arteries and veins in the aortoiliac vasculature, the CAA and CVA are determined from 
TABLE I

Maximum Mean Similarity $\left(S_{\max }\right)$ and Mean Area Under the Accuracy Plot $\left(A_{\text {acc }}\right)$ for the Forces. The Best Performing COMBINATION IS SHOWN IN BOLD

\begin{tabular}{|c|c|c|c|c|c|c|}
\hline$F_{\text {ext }}$ & $\epsilon^{1}$ & atc $^{2}$ & $S_{\max }$ & $A_{a c c}{ }^{3}$ & Best performing parameters & $\begin{array}{l}\text { Figure } 3 \\
\text { row }\end{array}$ \\
\hline$F_{\text {int }}$ & 0 & 0 & 0.93 & 0.96 & n.a. & 1 \\
\hline$F_{\text {int }}$ & 0 & 1 & 0.93 & 0.97 & n.a. & 1 \\
\hline$F_{\text {grad }}$ & 0 & 1 & 0.81 & 0.83 & $\left(\sigma_{\text {grad }}, c_{\text {grad }}\right)=(1.25,18)$ & 2 \\
\hline$F_{\text {vessel }}$ & 0 & 1 & 0.83 & 0.92 & $\left(\alpha, \beta, \gamma / I_{\max }\right)=(0.25,0.50,0.375)^{4}$ & 3 \\
\hline$F_{\text {int }} F_{\text {grad }}$ & 0 & 1 & 0.94 & 0.98 & $\left(\sigma_{\text {grad }}, c_{\text {grad }}\right)=(1.25,18)$ & 4 \\
\hline$F_{\text {int }} F_{\text {vessel }}$ & 0 & 1 & 0.94 & 0.98 & $\left(\alpha, \beta, \gamma / I_{\max }\right)=(0.25,0.50,0.375)^{4}$ & \\
\hline$F_{\text {grad }} F_{\text {vessel }}$ & 0 & 1 & 0.86 & 0.93 & $\begin{array}{l}\left(\sigma_{\text {grad }}, c_{\text {grad }}\right)=(1.25,18) \\
\left(\alpha, \beta, \gamma / I_{\text {max }}\right)=(0.25,0.50,0.375)^{4}\end{array}$ & \\
\hline$F_{\text {int }} F_{\text {grad }} F_{\text {vessel }}$ & 0 & 1 & 0.93 & 0.97 & $\begin{array}{l}\left(\sigma_{\text {grad }}, c_{\text {grad }}\right)=(1.25,18) \\
\left(\alpha, \beta, \gamma / I_{\max }\right)=(0.25,0.50,0.375)^{4}\end{array}$ & \\
\hline $\mathbf{F}_{\text {Int }} \mathbf{F}_{\text {grad }}$ & 1 & 1 & 0.94 & 0.98 & $\begin{array}{l}\left(\sigma_{\mathrm{grad}}, \mathbf{c}_{\mathbf{g r a d}}\right)=(1.25,24) \\
\epsilon^{*}=\mathbf{0 . 0 5}\end{array}$ & 4 \\
\hline \multicolumn{7}{|c|}{$0 / 1=$ without/with curvature. } \\
\hline \multicolumn{7}{|c|}{${ }^{2} 0 / 1=$ single (arterial) front or two competative fronts, also referred to as arrival-time correction (atc). } \\
\hline \multirow{2}{*}{\multicolumn{7}{|c|}{${ }^{3} A_{a c c}$ is determined for $F P F \in[0,1]$; according to our definition $T P F$ can be smaller than one, }} \\
\hline & & & & & & \\
\hline \multicolumn{7}{|c|}{${ }^{4} I_{\max }$ is the maximum grey value in the image. } \\
\hline
\end{tabular}

the steady-state dataset using supervision. Hereto, the vesselness image of the steady-state dataset is computed at 25 scales (exponentially increasing, $\sigma=0.4-13.0 \mathrm{~mm}$ ) and the MCP is determined. Appropriate values for $\alpha, \beta$, and $\gamma$ were determined in an earlier study [23].

To find the CAA, the MCP is determined between user-defined points in the source data of the steady state (average six points (range [4-12]) per dataset) using the reciprocal values of the vesselness image as cost function. Similarly, the CVA is found by determining the MCP between user-defined points in the source data of the steady state (average 17 points (range [14-22]) per dataset).

\section{B. Parameter Selection}

In this section, the results of the experiments as described in Section III-C are discussed. Equation (8) is implemented using a simple Euler forward scheme. The time-step size was set to $\Delta t=0.1$. A narrow band that only updates the points within six voxels on either side of the zero level set is used to speed up the evolution [27]. The narrow band is updated after every 15 iterations.

The results of the experiments for the various terms are listed in Table I. Below, the results are reported according to the scheme in Section III-C.

1) The arrival-time correction is effective when the propagating arterial and venous fronts meet after some propagation time, i.e., after the arterial front has leaked through the boundaries of the artery (vein) of interest. Therefore, only FPF is influenced by the arrival-time correction, and TPF will be unaffected. It can be seen from the top row of Fig. 3 that FPF is reduced significantly for $t>10$. Although the difference in area under the accuracy plots is very small (approximately 0.01 ), the influence is still noticeable, as can be seen from the final results (see Fig. 4). This is explained from the fact that, in Fig. 3, the average results are shown, and the effect of the arrival-time correction can be seen at the smaller (iliac) vessels, where arteries and veins are in close proximity of each other. Since these small vessels form only a small part of the complete vasculature, the effect of the arrival-time correction is difficult to observe from the accuracy plot.

2) The results for $F_{\text {ext }}=F_{\text {int }}, F_{\text {ext }}=F_{\text {grad }}$, and $F_{\text {ext }}=$ $F_{\text {vessel }}$ are summarized here. Note that the normalized area under the accuracy plot $\left(A_{\mathrm{acc}}\right)$ is determined for $F P F \in[0,1]$.

- For $F_{\text {ext }}=F_{\text {int }}$, the performance of the method is shown in the top row of Fig. 3. It can be seen that, for $t=10$, the maximum mean similarity $\left(S_{\max }\right)$ is 0.93 and hardly decreases for increasing iteration time, indicating stability. $A_{\text {acc }}$ is 0.97 .

- For $F_{\text {ext }}=F_{\text {grad }}$, best initial results were obtained for $\left(\sigma_{\text {grad }}, c_{\text {grad }}\right)=(1.25,16)$. Since performancedecreased for increasing values of $\sigma_{\text {grad }}$ and $c_{\text {grad }}$, we also tested $c_{\text {grad }} \in\{16,17, \ldots, 24\}$ and 

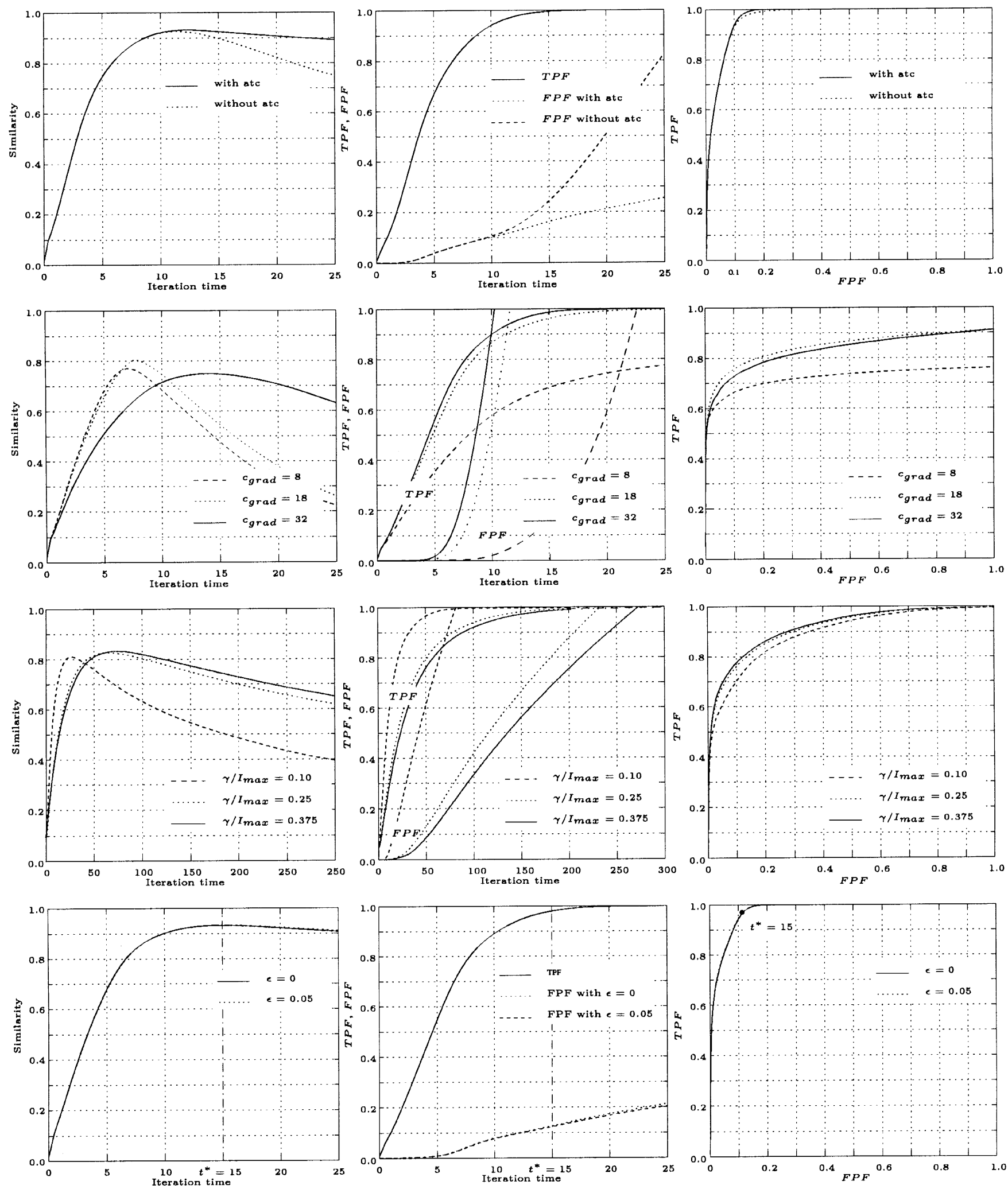

Fig. 3. Mean similarity $(S)$, mean false and true positive fraction ( $F P F$ and $T P F$, respectively), and mean accuracy plot of the LSB separation (left, middle, and right columns, respectively) for different combinations of $F_{\text {ext }}$ (atc). For all experiments, time-step size $\Delta t$ was set to 0.1 . (top row) $F_{\text {ext }}=F_{\text {int }}$, with and without atc, and $\epsilon=0$. Evolution of the arterial front only, is similar to not using the atc. (second row) $F_{\text {ext }}=F_{\text {grad }}$, with $c_{\text {grad }}=1.25$ and $c_{\text {grad }} \in\{8,18,32\}$, with atc, and $\epsilon=0$. Note that the $A_{\text {acc }}$ is determined for $F P F \in[0,1]$ even though $T P F<1$. (third row) $F_{\text {ext }}=F_{\text {vessel }}$, with $(\alpha, \beta)=(0.25,0.5)$ and $\gamma / I_{\max } \in\{0.10,0.25,0.375\}$ ( $I_{\max }$ is the maximum grey value in the image), with atc, and $\epsilon=0$. (bottom row) $F_{\text {ext }}^{*}=F_{\text {int }} F_{\text {grad }}$, with $\left(\sigma_{\text {grad }}, c_{\text {grad }}\right)=$ $(1.25,24)$, with atc, and $\epsilon \in\{0,0.05\}$.

$\sigma_{\text {grad }} \in\{1.0,1.25,1.5\}$. Best results $\left(S_{\max }=0.81\right.$ and $A_{\text {acc }}=0.83$ ) were obtained for $c_{\text {grad }}=18$, at which $\sigma_{\text {grad }}$ did not influence the results, indicating the robustness for variation in this parameter. In the 

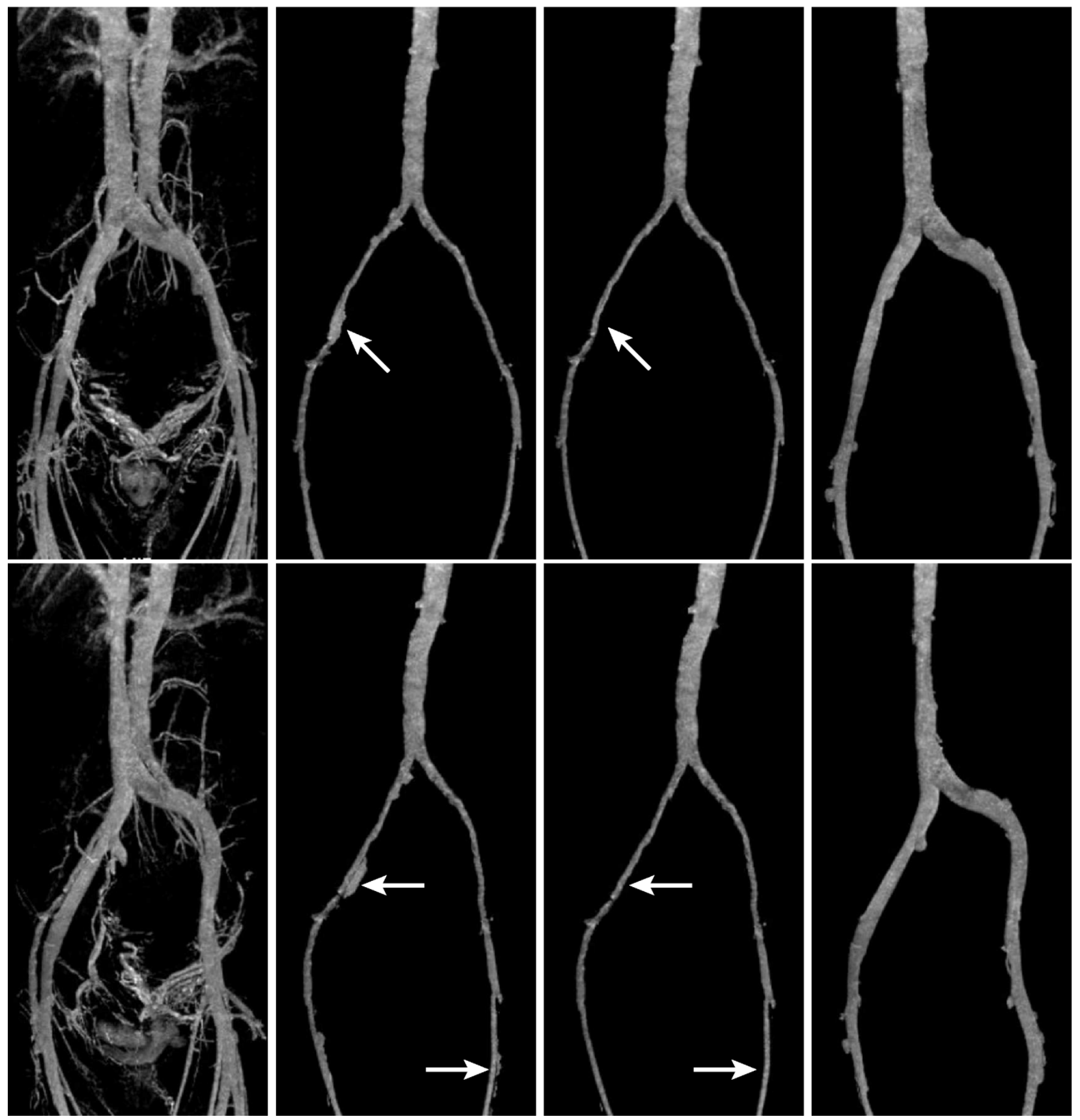

Fig. 4. MIP of a steady-state dataset (first column). It can be seen that arterial interpretation is hampered without AV separation, whereas after separation, a good overview of the main arteries and veins is achieved (remaining columns). In the top (bottom) row, posteroanterior (oblique) views are shown. If only the arterial front is used to segment the arteries (second column), leakage from the arterial front in the venous part of the vasculature is inevitable (at the arrows in the second column). However, this leakage can be corrected for if both the arterial and venous front are evolved. Based on the arrival time of these two fronts, voxels are labeled either arterial or venous. Separated arteries and veins using this arrival-time correction are shown in the third and fourth columns, respectively.

second row of Fig. 3, we show the performance of the method for $F_{\text {ext }}=F_{\text {grad }}$ with $\sigma_{\text {grad }}=1.25$ and $c_{\mathrm{grad}} \in\{8,18,32\}$. Results for other values of $c_{\text {grad }}$ and $\sigma_{\text {grad }}$ are not shown here, but similar plots are obtained. Note that FPF increases rapidly, which indicates that the front leaked through the vessel boundaries.
- For $F_{\text {ext }}=F_{\text {vessel }}$ with $(\alpha, \beta)=(0.25,0.5)$ and $\gamma / I_{\max } \in\{0.10,0.25,0.375\}$, the performance of the method is shown in the third row of Fig. 3. For $\gamma / I_{\max }=0.375$, we find the best results viz. $S_{\max }=0.83$ and $A_{\text {acc }}=0.92$. Note that for $F_{\text {ext }}=$ $F_{\text {vessel }}$ a much larger iteration time is needed in order to obtain the most accurate segmentation. 
After testing the individual terms, it can be concluded that $F_{\text {ext }}=F_{\text {int }}$ outperforms $F_{\text {ext }}=F_{\text {grad }}$ and $F_{\text {ext }}=$ $F_{\text {vessel }}$ both in performance and stability.

3) All combinations of the different terms are tested using the best parameter settings $\left(\sigma_{\text {grad }}, c_{\text {grad }}\right)=(1.25,18)$ and $\left(\alpha, \beta, \gamma / I_{\max }\right)=(0.25,0.5,0.375)$. The combination $F_{\text {ext }}=F_{\text {int }} F_{\text {grad }}$ yielded the best results. To determine whether the performance of this force could improve for other values of the parameters, we also tested this combination for $\sigma_{\text {grad }} \in\{1.0,1.25,1.5\}$ and $c_{\text {grad }} \in\{16,18, \ldots, 30,32\}$. Best results ( $S_{\max }=0.94$ and $A_{\mathrm{acc}}=0.98$ ) were obtained for $c_{\text {grad }} \in\{20, \ldots, 28\}$, while $\sigma_{\text {grad }}$ did not influence these results.

Two conclusions can be drawn from these results: first, $F_{\text {int }}$ is predominant in the combination $F_{\text {ext }}=F_{\text {int }} F_{\text {grad }}$, which was to be expected based on the results under 1) and 2). Second, for the combination $F_{\text {ext }}=F_{\text {int }} F_{\text {grad }}$, the performance is hardly affected by $\sigma_{\text {grad }}$ and $c_{\text {grad }}$ (on the intervals mentioned above), indicating the robustness for variation in these parameters. For further experiments, we chose $\left(\sigma_{\text {grad }}, c_{\text {grad }}\right)=(1.25,24)$ as the best parameters for $F_{\text {ext }}^{*}=F_{\text {int }} F_{\text {grad }}$.

4) The influence of curvature is tested using $F_{\text {ext }}^{*}=$ $F_{\text {int }} F_{\text {grad }}$ with $\left(\sigma_{\text {grad }}, c_{\text {grad }}\right)=(1.25,24)$. Curvature did not influence the results significantly: $S_{\max }=0.94$ and $A_{\text {acc }}=0.98$. However, for $\epsilon^{*}=0.05, F P F(t)$ is smallest (see bottom row of Fig. 3).

5) From seven patient datasets, arteries and veins were separated. Hereto, we applied the best performing image-based force $F_{\text {ext }}^{*}=F_{\text {int }} F_{\text {grad }}$ and curvature weighting factor $\epsilon^{*}=0.05$. To determine the best performing number of iterations, we selected a cut point $\left(t^{*}\right)$ using the bottom row of Fig. 3 : for $t^{*}=15$, we find $T P F=0.98, F P F=0.13$, and similarity is (and remains almost) maximal for increasing $t$.

In Figs. 4 and 5, the results are shown for two different subjects. In all datasets, a straightforward MIP (and even a targeted MIP) can hardly be interpreted owing to venous overlap. Using the automatically obtained central axes, the complete arterial (venous) vasculature can be inspected without venous (arterial) contamination, thus providing the clinicians with relevant diagnostic information on just a few mouse clicks. In the arterial segmentation in Fig. 5, a stenosis is visible, which cannot be detected in the corresponding MIPs of the steady-state dataset.

\section{DISCUSSION}

Improved visualization, for example, by means of $\mathrm{AV}$ separation, is the key issue that needs to be solved for BPAs to gain clinical acceptance in MRA. Several authors proposed techniques for AV separation. These techniques can mainly to be subdivided into two categories, viz. separation during the acquisition stage and separation in the post-processing stage. In this paper, we have presented an LSB technique for segmentation of the aortoiliac vasculature, which falls in the second category.
The novelty of this approach is twofold.

- The central arterial axes and central venous axes are used for the initialization of the LSB segmentation. Manually outlining the CAA and CVA in the steady-state dataset is a tedious procedure that cannot be used in clinical practice. Therefore, we have applied a supervised method for the determination of these central axes, which requires only minimal user interaction. By utilizing a central axis as initialization, we assure a good initialization for the level-set approach, also when a stenosis is present. Furthermore, the central axes ensure initialization that is everywhere close to the vessel wall. If only one single point or a more local initialization is used, leakage may occur near the initialization before the vessel of interest is segmented. The amount of user interaction can be further reduced if a first-pass dataset (in which only the arterial part of the vasculature is enhanced) is available. Only three user-defined points are needed to determine the CAA if a first-pass dataset is available [23]. Although Lei et al. have used central axes as initialization (see [20] for fuzzy connectedness AV separation), the use of central axes as initialization for evolving level sets has not been reported earlier.

- The presented approach is based on the simultaneous propagation of two competitive fronts representing the evolving arterial and venous segmentation. The evolution of these fronts is started from the CAA and CVA in the steady-state dataset, respectively. Since arteries and veins can be in close proximity of each other, leakage from the evolving arterial (venous) front into the venous (arterial) part of the vasculature is inevitable. In these situations, AV separation is achieved by labeling voxels arterial or venous based on the arrival time of the respective front. It has been shown that the arrival-time correction is effective, especially at the iliac arteries. Lei et al. [20] performed a similar AV separation based on relative fuzzy connectedness.

Parameter optimization and accuracy assessment of the method is achieved by comparing the LSB segmentations with the reference standard, i.e., manually obtained segmentations. Owing to the enhancement of the entire vascular structure in the steady state, it is not feasible to perform a complete manual segmentation of the aortoiliac vasculature. Therefore, representative subvolumes are used for which manual segmentation of the arteries is a feasible task. In LSB segmentation, both the initialization and applied force play a key role. The influence of three image-based forces has been tested. Finally, the best performing force is applied to seven patient datasets for $\mathrm{AV}$ separation. Using the CAA and CVA as initialization, the actual separation of arteries and veins could be achieved with these fixed parameter settings without any further user interaction. Improved visualization of separated arteries and veins could, therefore, be achieved. Higher order branches can be separated, but more user interaction is needed.

In the proposed method for AV separation of the aortoiliac vasculature, the preprocessing, i.e., vessel-enhancement filtering, is most time consuming. All patient datasets could be cropped from $512 \times 512 \times 100$ to a FOV of $325 \times 512 \times 85$. The images were filtered at 25 scales in approximately $25 \mathrm{~min}$ on 

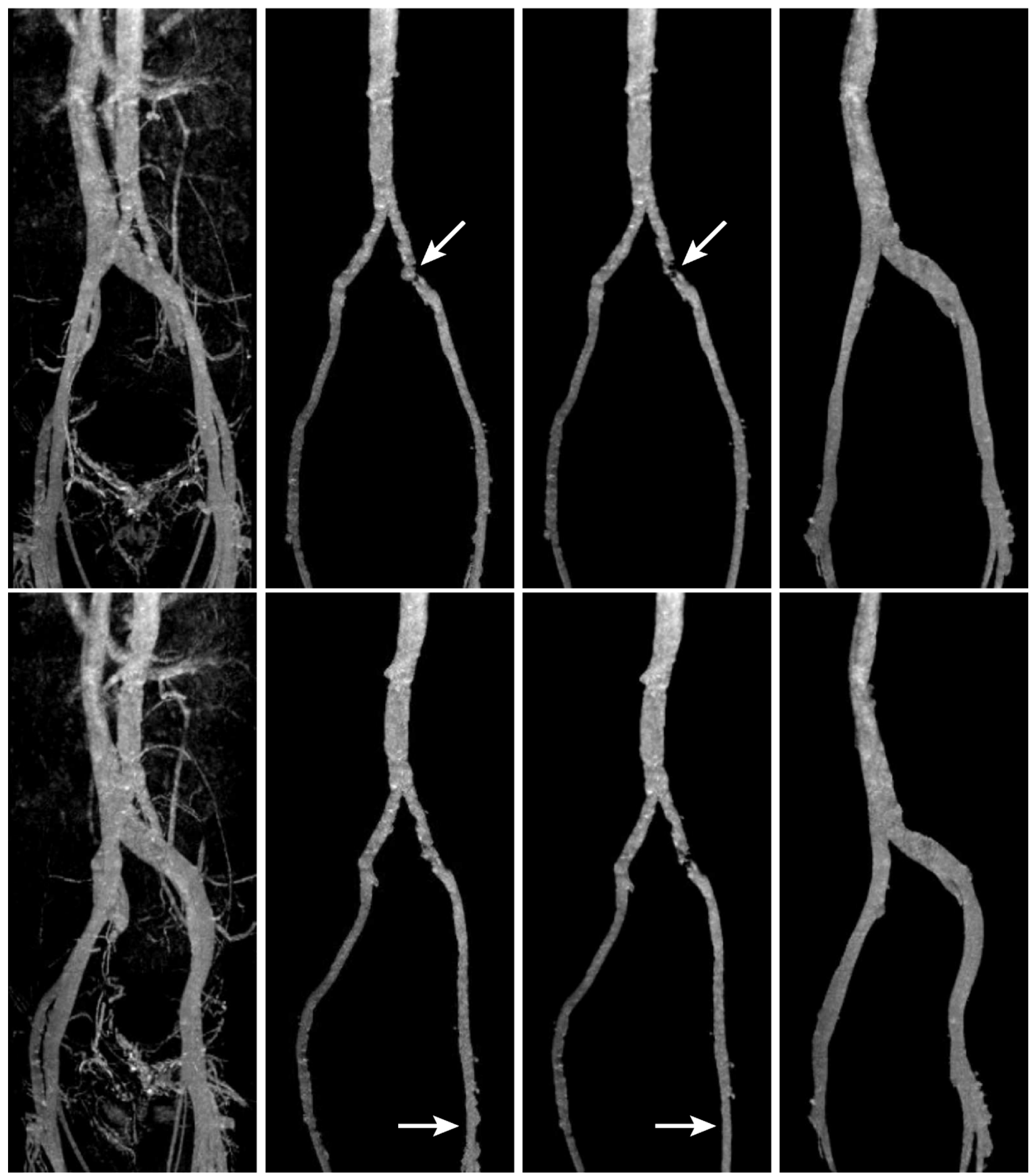

Fig. 5. MIP of a steady-state dataset (first column). It can be seen that arterial interpretation is hampered without AV separation, whereas after separation, a good overview of the main arteries and veins is achieved (remaining columns). In the top (bottom) row, posteroanterior (oblique) views are shown. If only the arterial front is used to segment the arteries (second column), leakage from the arterial front in the venous part of the vasculature is inevitable (at the arrows in the second column). However, this leakage can be corrected for if both the arterial and venous front are evolved. Based on the arrival time of these two fronts, voxels are labeled either arterial or venous. Separated arteries and veins using this arrival-time correction are shown in the third and fourth columns, respectively. In the top row, a stenosis is present at the location of the arrow. This stenosis could not be detected in the MIP of the whole steady-state dataset. Both the original dataset and the MIPs of the arterial segmentations were inspected by a radiologist, who judged that the MIP of the arterial segmentation with arrival-time correction was better.

a UNIX workstation (UltraSPARC-III, 900-MHz processor). The number of scales can be reduced since $F_{\text {vessel }}$ is used in the
CAA and CVA extraction and is not used in the AV separation. The actual AV separation can be performed in approximately 
15 min. We believe that the presented method is an important step toward improved interpretation of steady-state BPAs.

\section{REFERENCES}

[1] T. M. Grist, F. R. Korosec, D. C. Peters, R. C. Walovitch, R. P. Dolan, W. E. Bridson, E. K. Yucel, and C. A. Mistretta, "Steady-state and dynamic MR angiography with MS-325: Initial experience in humans," Radiology, vol. 207, pp. 539-544, 1998.

[2] M. Saeed, M. F. Wendland, and C. B. Higgins, "Blood pool MR contrast agents for cardiovascular imaging," J. Magn. Reson. Imag., vol. 12, pp. $890-898,2000$

[3] D. A. Bluemke, R. D. Darrow, R. Gupta, S. K. Tadikonda, and C. L. Domoulin, "3D contrast enhanced phase contrast angiography: Utility for arterial/venous segmentation," in Proc. Int. Soc. Magnetic Resonance in Medicine, vol. 7, 1999, p. 1237.

[4] T. K. F. Foo, V. B. Ho, M. N. Hood, J. M. Czum, S. D. Wolff, Y. Zhang, and P. L. Choyke, "A novel method for MR arterial and venous discrimination using gated phase contrast and VENC selection," in Proc. Int. Soc. Magnetic Resonance in Medicine, vol. 7, 1999, p. 2182.

[5] Y. Wang, Y. Yu, D. Li, K. T. Bae, J. J. Brown, W. Lin, and E. M. Haacke, "Artery and vein separation using susceptibility-dependent phase in contrast-enhanced MRA," J. Magn. Reson. Imag., vol. 12, pp. 661-670, 2000.

[6] J. Svensson, P. Leander, J. H. Maki, F. Stahlberg, and L. E. Olsson, "Separation of arteries and veins using flow-induced phase effects in contrast-enhanced MRA of the lower extremities," J. Magn. Reson. Imag., vol. 20, pp. 49-57, 2002.

[7] Y. Mazaheri, T. J. Carroll, J. Du, W. F. Block, S. B. Fain, T. F. Hany, B. D. L. Aagaard, C. M. Strother, C. A. Mistretta, and T. M. Grist, "Combined time-resolved and high-spatial-resolution 3D MRA using an extended adaptive acquisition," J. Magn. Reson. Imag., vol. 15, pp. 291-301, 2002.

[8] C. Lorenz, I.-C. Carlsen, T. M. Buzug, C. Fassnacht, and J. Weese, "Multi-scale line segmentation with automatic estimation of width, contrast and tangential direction in 2D and 3D medical images," in Proceedings of Computer Vision, Virtual Reality and Robotics in Medicine (CVRMed) and Medical Robotics and Computer Assisted Surgery (MRCAS), J. Troccaz, E. Grimson, and R. Mösgez, Eds. Berlin, Germany: Springer-Verlag, 1997, Lecture Notes Comput. Sci., pp. 233-242.

[9] Y. Sato, S. Nakajima, H. Atsumi, T. Koller, G. Gerig, S. Yoshida, and R. Kikinis, "3D multi-scale line filter for segmentation and visualization of curvilinear structures in medical images," in Proceedings of Computer Vision, Virtual Reality and Robotics in Medicine (CVRMed) and Medical Robotics and Computer Assisted Surgery (MRCAS), J. Troccaz, E. Grimson, and R. Mösgez, Eds. Berlin, Germany: Springer-Verlag, 1997, Lecture Notes Comput. Sci. 1205, pp. 213-222.

[10] A. F. Frangi, W. J. Niessen, K. L. Vincken, and M. A. Viergever, "Multiscale vessel enhancement filtering," in Proceedings of Medical Image Computing and Computer-Assisted Intervention, W. M. Wells, A. Colchester, and S. Delp, Eds. Berlin, Germany: Springer-Verlag, 1998, Lecture Notes Comput. Sci., pp. 130-137.

[11] L. M. Lorigo, O. Faugeras, W. E. L. Grimson, R. Keriven, R. Kikinis, A. Nabavi, and C.-F. Westin, "CURVES: Curve evolution for vessel segmentation," Med. Image Anal., vol. 5, pp. 195-206, 2001.
[12] I. Nyström and Ö. Smedby, "Skeletonization of volumetric vascular image distance information utilized for visualization," J. Combinatorial Optimization, vol. 5, no. 1, pp. 27-41, 2001.

[13] S. Aylward and E. Bullitt, "Initialization, noise, singularities, and scale in height ridge traversal for tubular object centerline extraction," IEEE Trans. Med. Imag., vol. 21, pp. 61-75, Feb. 2002.

[14] J. S. Suri, K. Liu, L. Reden, and S. Laxminarayan, "A review on MR vascular image processing algorithms: Acquisition and prefiltering: Part I," IEEE Trans. Inform. Technol. Biomed., vol. 6, pp. 324-337, Dec. 2002.

[15] — - "A review on MR vascular image processing algorithms: Skeleton versus nonskeleton approaches: Part II," IEEE Trans. Inform. Technol. Biomed., vol. 6, pp. 338-350, Dec. 2002.

[16] M. Bock, S. O. Schoenberg, F. Floemer, and L. R. Schad, "Separation of arteries and veins in 3D MR angiography using correlation analysis," Magn. Reson. Med., vol. 43, pp. 481-487, 2000.

[17] W. J. Niessen, A. D. Montauban van Swijndregt, B. H. P. Elsman, O. Wink, M. A. Viergever, and W. P. T. M. Mali, "Enhanced artery visualization in blood pool MRA: Results in the peripheral vasculature," in Proceedings of Medical Image Computing and Computer-Assisted Intervention, W. M. Wells, A. Colchester, and S. Delp, Eds. Berlin, Germany: Springer-Verlag, 1998, Lecture Notes Comput. Sci., pp. 340-345.

[18] R. M. Stefancik and M. Sonka, "Highly automated segmenation of arterial and venous trees from three-dimensional magnetic resonance angiography (MRA)," Int. J. Cardiovasc. Imag., vol. 17, pp. 37-47, 2001.

[19] X. Tizon and Ö. Smedby, "Segmentation with gray-scale connectedness can separate arteries and veins in MRA," J. Magn. Reson. Imag., vol. 15, pp. 438-445, 2002.

[20] T. Lei, J. K. Udupa, P. K. Saha, and D. Odhner, "Artery-vein separation via MRA-An image processing approach," IEEE Trans. Med. Imag., vol. 20, pp. 689-703, Aug. 2001.

[21] J. K. Udupa and S. Samarasekera, "Fuzzy connectedness and object delineation: Theory, algorithm, and validation," Graph. Models Image Process., vol. 58, pp. 246-261, 1996.

[22] O. Wink, W. J. Niessen, and M. A. Viergever, "Minimum cost path determination using a simple heuristic function," in Proceedings of the International Conference on Pattern Recognition, A. Sanfelin, J. J. Villanueva, M. Vanrell, R. Alquézar, T. Huang, and J. Serra, Eds. Piscataway, NJ: IEEE Computer Soc., 2000, pp. 1010-1013.

[23] C. M. van Bemmel, W. J. Niessen, O. Wink, B. Verdonck, and M. A. Viergever, "Blood pool contrast-enhanced MRA: Improved arterial visualization in the steady state," IEEE Trans. Med. Imag., vol. 22, no. May, pp. 645-653, 2003.

[24] J. A. Sethian, Level Set Methods and Fast Marching Methods, 2nd ed. Cambridge, U.K.: Cambridge Univ. Press, 1999.

[25] M. Kass, A. P. Witkin, and D. Terzopoulos, "Snakes: Active contour models," Int. J. Comput. Vis., vol. 1, no. 4, pp. 321-331, 1988

[26] L. Ambrosio and H. M. Soner, "Level set approach to mean curvature flow in arbitrary codimension," J. Differ. Geometry, vol. 43, pp. 693-737, 1996.

[27] R. Malladi, J. A. Sethian, and B. C. Vemuri, "Shape modeling with front propagation: A level set approach," IEEE Trans. Pattern Anal. Machine Intell., vol. 17, pp. 158-175, Feb. 1995.

[28] C. M. Bishop, Neural Networks for Pattern Recognition. New York: Oxford Univ. Press, 1995.

[29] B. N. Joe, M. B. Fukui, C. C. Meltzer, Q. Huang, R. S. Day, P. J. Greer, and M. E. Bozik, "Brain tumor volume measurement: Comparison of manual and semiautomated methods," Radiology, vol. 212, no. 3, pp. 811-816, 1999. 